\title{
Knowledge, attitude and practices related to Brucellosis in the people of Khyber Pukhtun Khwa Province, Pakistan
}

\author{
Muhammad Khan ${ }^{1}$, Zhijun $\mathrm{Cao}^{1}$, Adnan Khan ${ }^{1}$, Sumerana Kausar ${ }^{2}$, Jianxin Xiao ${ }^{1}$, and \\ Naeem Ud-din ${ }^{3}$ \\ ${ }^{1}$ China Agricultural University College of Animal Science and Technology \\ ${ }^{2}$ Beijing Forestry University \\ ${ }^{3}$ Livestock department, Khyberpukhtun Khwa, Pakistan
}

July 9, 2020

\begin{abstract}
Objectives: The current study was carried out to explore the knowledge, attitude, and practices towards Brucellosis in the people of Khyber Pukhtun Khwa Province, Pakistan. Study design: Cross-sectional study Material and methods: Total of 1600 participants belong to different professions, education level, locality and nature of contact with animals were selected by random sample method. The samples were selected from the Khyber Pakhtun Khwa province of Pakistan. Results: The findings of this study documented poor knowledge regarding brucellosis in the selected participants of current study. Although human and animal health professionals had sufficient knowledge about brucellosis, however no serious trends to control brucellosis were noticed even in health professionals. The unhygienic approach was observed in farmers regarding the handling animal and their products. Self medication approach was also documented in many participants which create antibiotic resistance. Conclusions: Based on the findings we noticed a lack of information regarding zoonotic diseases, health facilities and a weak bond between health and non-health professionals in KPK. Proper education and awareness is highly recommended on zoonotic diseases. Keywords: Zoonotic diseases, Brucellosis, Knowledge, Practices, Attitude
\end{abstract}

\section{Keywords: Zoonotic diseases, Brucellosis, Knowledge, Practices, Attitude}

\section{Introduction}

Brucellosis is a contagious and highly zoonotic disease which is caused by Brucella species. ${ }^{1}$ Annually approximately 500,000 cases of brucellosis have been reported in a study and considered as a serious threat to public health. ${ }^{2,3}$ Human gets the infection when coming in contacts with brucella-infected animals or by using their by-products such as meat and milk. Besides, the disease is a big challenge for the economy of a country as well. ${ }^{4}$ The main clinical signs in of brucellosis in cattle are undulant fever, abortion in the last trimester, and swelling of udder up-to-the naval region and sterility. ${ }^{5}$ In human, the disease brucellosis is also called undulant fever, which is characterized by septicemia, followed by high fever, abortion, emaciation, and arthritis. ${ }^{6}$ Although livestock is one of the essential assets of low-income people to meet their livelihood needs, ${ }^{7}$ however, animal diseases including zoonoses, are the critical limitations enhanced due to livestockproduction systems. ${ }^{8}$ Brucellosis is an expanding infectious and zoonotic disease with increasing human and animal health impact in Pakistan. The majority of the people in rural areas depend on livestock for income; however, most of the people have not knowledge about the serious consequences of brucellosis. The purpose of this study was to evaluate the attitude and knowledge towards brucellosis in the people of KPK province, Pakistan.

\section{Methods}




\subsection{Study area, design and sample population}

The cross-sectional study was carried out in Khyber Pukhtun Khwa Province, Pakistan. In total, 1600 samples were selected by random sampling method. The aims of the study were explained to the participants in three languages namely Pashtu, Urdu and English. The participants were informed about the implication of the study and were guaranteed the privacy of the information taken from them. The first part of the questionnaire was related to the occupation, education level and locality, while the second portion contained the questions associated with the Knowledge about Brucellosis (Zoonotic importance, clinical signs, and way of transmission) practices and attitude towards Brucellosis. Descriptive type statistical analyses were carried to find out the percentage of participants against each question.

\section{Results}

\subsection{Knowledge of Brucellosis}

Different questions related to knowledge on Brucellosis were asked from the participants. Out of 1600 participants, only $25 \%$ had knowledge about zoonosis. Similarly, $9.38 \%, 6.25 \%, 5 \%$ of respondents answered yes against the questions knowledge about brucellosis, clinical signs, transmission to human and animals which can transmit brucellosis to human respectively (Table 1). In addition, the main sources of information regarding brucellosis were Academic (25\%), newspaper (6.25\%), and electronic media (12.5\%). When the question regarding the use of infected animals' by-product (milk/meat) was asked, $91.25 \%$ of precipitants replied in "I don't know". The details of the results are summerized in Table 1

\subsection{Attitude toward Brucellosis}

Three questions, including treatment strategy of brucellosis, dealing with infected and died animal due brucellosis were asked. The response of participants was $12.5 \%, 44 \%, 18.75 \%$, and $25 \%$ for questions associated with self-medication, consult doctor, damn care and not sure respectively. Furthermore, the high percentages (50\%) of participants were in the favour to sell the Brucella infected animals, while $25 \%$ were thinking that the byproduct of Brucella infected animals can be used. It was also noticed that the majority of the participants (46.88\%) recommended that the animals died due to Brucella can be throw in an open field, while $40.62 \%$ of respondents were placed in the category who were not sure how to deal with these animals (table 1).

\subsection{Practices toward Brucellosis}

For practices, $62.25 \%$ of respondents were placed in the group which practices regular hand washing after in contact with animals directly or by using their by-products. The rest of $37.5 \%$ were not caring too much about hand washing after handling animals. $56.25 \%$ of participants answered that they drink raw milk. Regarding the test for Brucellosis, most of the respondents were not testing their animals for Brucellosis and $62.25 \%$ ware placed in the group, which was in close proximity with animals (Table 1).

\section{Discussion}

We evaluated the knowledge, attitude and practices relating to Brucellosis in KPK, Pakistan. The findings of the current study showed that most of the participants are not aware of the transmission, treatment and hygienic measures of brucellosis. Furthermore, it was observed that most of the veterinary professional had a deep knowledge about brucellosis transmission, treatment and prevention strategies. However, poor trends of attitude, and practices regarding brucellosis in the current group of people selected for this study. It was indeed a matter of attention because the major group of people having even direct contact with the animal still had no or poor knowledge about zoonotic diseases including brucellosis. Self-medication inclination was noticed in the majority of participants. Self-medication is not only dangerous for the users himself but also a threat to the globe. Because of self-medication, bacterial resistance creates which is another burning issue not only facing Pakistan but also the globe. The farmers in villages have limited or no access to the animal health related facilities in the majority of areas of KPK, Pakistan. The other reasons for the above shortcomings were lack of scientific knowledge, communication gap among Veterinarians, human 
health professionals and common people of the community. Even though Pakistan is an agricultural country where most of the people practices livestock, however, to date, not a single centre for zoonotic diseases has been established in Pakistan.

\section{Recommendations}

The current study showed that overall the participants had lack of information regarding brucellosis. Thus particular emphasis is therefore required in the rural areas of Khyber Pakhtun Khwa, where the general public should be informed on the issues of brucellosis, and cattle handlers, especially farmers should be equipped with the requisite facilities, protective measures and preventive assistance. Additionally, systematic supervision is suggested to make sure that all these actions and services are utilized effectively and that the aim of managing and avoiding the disease is accomplished. Brucellosis is a serious problem, and a comprehensive multidisciplinary initiative is required to cope with the situation before it becomes an epidemic. In addition, an emphasis on vertical programs for the management of zoonotic diseases is required. Strong bond among health departments, teamwork with World Organization for Animal Health (OIE), implementation and monitoring of policies for the effective measure are highly encouraged. Development of collaboration among animal health researchers and instructive campaigns that focus general public or animal handlers in rural areas are suggested. Furthermore, collaborative work among endemic nations will further build up a strong response against brucellosis.

\section{Authors' Statement}

MZK and ZC supervised and designed the study. MZK wrote the manuscript. ZC and AK edit the manuscript. AK, MZK, SK, JX and NUD collected data.

\section{Acknowledgments}

We are thankful to the College of Animal Science and Technology, China Agricultural University, Beijing, China, for providing us an environment of learning. Without their platform, the completion of this work was not an easy task. Besides, we would like to thank all the participants who participated in the survey.

\section{Ethical approval}

No ethical approval was required as it was a survey based study; however, the data were collected after obtaining consent from all the participants involved in the study. In addition, the questionnaire was anonymous, with no name or identity. The survey did not include any sensitive issues.

\section{Funding}

The study received no funding.

\section{Competing interests}

The authors declare that they have no competing interests.

\section{Data Availability}

The data is already available in main manuscript

\section{References}

1. Godfroid J. Brucellosis in livestock and wildlife: zoonotic diseases without pandemic potential in need of innovative one health approaches. Arch Public Health 2017; 75: 34.

2. Pappas G, Papadimitriou P, Akritidis N, Christou L, Tsianos EV. The new global map of human Brucellosis. Lancet Infect Dis 2006; 6(2):91-99.

3. Seleem, MN, Boyle SM, Sriranganathan N. Brucellosis: a re-emerging zoonosis. Vet Microbiol 2010; 140(3-4): 392-398.

4. Singh BB, Khatkar MS, Aulakh RS, Gill JPS, Dhand NK. Estimation of the health and economic burden of human Brucellosis in India. Prev Vet Med 2018; 154: 148-155. 
5. Khan ZM and Zahoor M. An Overview of Brucellosis in Cattle and Humans, and its Serological and Molecular Diagnosis in Control Strategies. Trop Med Infect Dis 2018; 3:65

6. Franco, M.P., Mulder, M., Gilman, R.H., Smits, H.L., 2007. Human Brucellosis. Lancet Infect Dis 2007; 7:775-786.

7. Perry, B., Grace, D., 2009. The impacts of livestock diseases and their control on growth and development processes that are pro-poor. Philos. Trans. R Soc. Lond B Biol Sci 2009; 364 (1530):2643-2655.

8. FAO. Socio-economic consequences for poor livestock farmers of animal diseases and VPH problems (chapter 1). Improved Animal Health for Poverty Reduction and Sustainable Livelihoods. FAO Animal Production and Health Paper 153, 2002. Published by FAO, Rome 2002.

\section{Hosted file}

table. docx available at https://authorea.com/users/341346/articles/468280-knowledge-attitudeand-practices-related-to-brucellosis-in-the-people-of-khyber-pukhtun-khwa-provincepakistan 